\title{
Genetic polymorphisms of CYP2D6 oxidation in patients with systemic lupus erythematosus
}

Jadwiga Skrętkowicz ${ }^{1}$, Małgorzata Barańska ${ }^{1}$, Anna Kaczorowska², Mariola Rychlik-Sych ${ }^{1}$

1Department of Pharmacogenetics, Medical University of Lodz, Poland

2Department of Dermatology and Paediatric Dermatology, Military-Medical Faculty, Medical University of Lodz, Poland

Submitted: 25 May 2010

Accepted: 26 August 2010

Arch Med Sci 2011; 7, 5: 864-869

DOI: $10.5114 /$ aoms.2011.25563

Copyright (c) 2011 Termedia \& Banach

\section{Abstract}

Introduction: Systemic lupus erythematosus (SLE) is a complex, multifactor autoimmune disease. The studies on aetiopathogenesis of autoimmune diseases focus on the impact the genetically conditioned impairment of xenobiotic metabolism may exert. The knowledge of oxidation polymorphism in the course of SLE may be helpful in choosing more efficient and safer therapy. We determined whether there was an association between susceptibility to SLE and particularly to CYP2D6 genotypes.

Material and methods: The study was carried out in 60 patients with SLE and 129 healthy volunteers and all the subjects were of Polish origin. The samples were analysed for two major defective alles for CYP2D $-C Y P 2 D 6^{\star} 3$ and CYP2D $6^{*} 4$ and one wild -type allele CYP2D6* 1 -by the polymerase chain reaction fragment length polymorphism (PCR-RFLP) metod with DNA extracted from peripheral blood.

Results: No statistically significant differences in the incidence of CYP2D6 genotypes between the studied groups were found $(p=0.615)$. Risk $(\mathrm{OR})$ of SLE development was 1.03 for the carriers of CYP2D $6^{*} 3$ allele and 1.48 for the subjects with $C Y P 2 D 6^{*} 4$ allele; but it was not statistically significant.

Conclusions: Increased occurrence of mutant alleles of the CYP2D6 gene in SLE patients and the calculated OR values could suggest the effect of these mutations on increased SLE development.

Key words: genetic polymorphism, oxidation, systemic lupus erythematosus.

\section{Introduction}

In recent years, numerous observations have elucidated the role of hereditary factors in susceptibility to some diseases. Systemic lupus erythematosus (SLE) is one of them.

Systemic lupus erythematosus is a chronic, systemic autoimmune disease with a complex pathogenesis involving multiple genetic and environmental factors. Systemic lupus erythematosus predominantly affects women in childbearing age. The disease is characterized by autoantibody production, abnormalities in the immune-inflammatory system function and inflammatory manifestation in several organs [1]. The aetiology of SLE is still unclear. The pathogenic process involves a complex interaction between environmental agents, hormones, immunomodulatory factors and disease susceptibility genes that predispose to SLE. The disease becomes symptomatic when the total of effects of different factors exceeds

\author{
Corresponding author: \\ Małgorzata Barańska MD, \\ PhD \\ Department of \\ Pharmacogenetics \\ Medical University of Lodz \\ 1 Muszyńskiego \\ 90-151 Lodz, Poland \\ Phone: +48426779177 \\ Fax: +48426788398 \\ E-mail: \\ malgorzata.baranska@ \\ umed.lodz.pl
}


a certain threshold, which causes an improper production of autoantibodies [2]. The genetic background of SLE is thought to be complex and involves multiple genes encoding different molecules with significant functions in immune system regulation [1, 3-5]. The group of candidate genes includes those encoding the activity of enzymes that metabolise xenobiotics. Environmental factors have been known to play a crucial role in autoimmune diseases; therefore numerous studies are being performed whose aim is to elucidate the effect of xenobiotics on the occurrence of autoimmune processes leading to SLE development. Many xenobiotics may induce alterations in the chromosomal structure, e.g., drugs, pesticides, fungicides, or substances causing environmental pollution [6]. The studies on aetiopathogenesis of autoimmune diseases focus on the impact the genetically conditioned impairment of xenobiotic metabolism may exert.

Many of these enzymes, including CYP2D6, play an instrumental role in the oxidation of multiple drugs and in the activation and deactivation of environmental xenobiotics such as carcinogens and toxic substances [6-8]. The CYP2D6 gene is localized on chromosome 22q13.1 [9]. The CYP2D6 (also called debrisoquine/sparteine hydroxylase) polymorphism was described in the 1970s while studying the metabolism of debrisoquine and sparteine [10]. Two phenotypically distinct groups were distinguished based on the capability to oxidise debrisoquine or sparteine to hydroxymetabolites in the human population: persons actively oxidizing the above-mentioned compounds - extensive metabolisers (EM) - and subjects who oxidize poorly, to a minimum degree - poor metabolisers (PM). In general, PM patients are characterized by reduced or no metabolic activity of CYP2D6 enzyme compared to EM patients, who have normal metabolic activity of CYP2D6 [11]. The polymorphism of the CYP2D6 gene has been relatively well explained, and more then 90 allelic variants of the CYP2D6 gene have been described. (http://www.imm.ki.se/CYPalleles). These variants result from point mutations, deletions or additions, gene rearrangements and deletion or duplication of the entire gene and result in an increase, reduction, or a complete loss of activity [6]. In general, $71 \%$ of CYP2D6 alleles in Caucasians are functional alleles, while non-functional alleles account for $26 \%$. The non-functional CYP2D6*4 (allele frequency $20 \%$ ) is carried by $75 \%$ of CYP2D6 poor metabolizers, while CYP2D6*3 is carried by $1-2 \%$ of them $[12,13]$. The polymorphisms of the CYP2D6 gene can result in defective or increased enzyme activity and CYP2D6 genotypes usually exhibit large inter-ethnic differences [8]. The knowledge of oxidation polymorphism in the course of SLE may be helpful in choosing more efficient and safer therapy, particularly in the case of a disease involving various organs and treated with drugs belonging to diverse therapeutic groups.

Studying gene-environment interactions in relation to the risk of SLE may be valuable because positive findings would clearly implicate the disease-causing exposures, clarify SLE aetiology and point to environmental modifications for disease prevention.

Despite the important role that CYP2D6 plays in detoxifying xenobiotics, few studies have investigated its function in SLE pathology $[14,15]$. Our study is the first that estimates the distribution of CYP2D6 genotypes in patients suffering from systemic lupus erythematosus from Poland.

The aim of our study was to analyse the CYP2D6 alleles, to assess the distribution of the genotypes in 60 patients with SLE and to establish whether there is any correlation between CYP2D6 genotype and SLE development.

\section{Material and methods}

The study was carried out in 60 patients with SLE diagnosed according to the criteria of the American College of Rheumatology [16]. The group of patients with SLE consisted of 54 women and 6 men (age range 17-76 years, mean $42 \pm 12.48$ years).The patients were hospitalized at the Department of Dermatology and Paediatric Dermatology, Military-Medical Faculty, Medical University of Lodz, Poland, and all the subjects were of Polish origin. The control group consisted of 129 volunteers (80 women and 49 men) aged 18 -73 years (mean age $41.3 \pm 15.41$ years) with no acute or chronic autoimmune diseases. The local ethics committee on human research approved the study, and informed consent was obtained from all patients.

The samples were analysed for two major defective alleles for CYP2D6 - CYP2D6*3 and CYP2D $6^{*} 4-$ and one wild-type allele $C Y P 2 D 6^{\star} 1$ by the polymerase chain reaction fragment length polymorphism (PCR-RFLP) method according to the procedure proposed by Smith et al. [17]. The application of the PCR-RFLP method allowed us to isolate two phenotypically distinct oxidation groups of subjects, i.e. poor metabolizers (PM), homozygotes possessing two mutated alleles: CYP2D $6^{*} 3$ and CYP2D6*4; extensive metabolizers (EM), heterozygotes possessing one wild-type allele $\left(C Y P 2 D 6^{*} 1\right)$ and one mutated allele (CYP2D6*3, CYP2D6 $\left.{ }^{\star} 4\right)$; and homozygotes possessing two wild alleles $\left(C Y P 2 D 6^{\star} 1\right)$ in their loci.

Genomic DNA was isolated from peripheral blood leukocytes. A $10 \mathrm{ml}$ venous blood sample was obtained from each patient and control and was stored in EDTA blood containers at $-20^{\circ} \mathrm{C}$. Genomic 
Table I. Distribution of poor and extensive genotypes in patients with SLE and in the controls

\begin{tabular}{|lccc|}
\hline Group & $\begin{array}{c}\text { Number } \\
\text { of patients }\end{array}$ & $\begin{array}{c}\text { Poor } \\
\text { metabolizers } \\
(\mathrm{PM})\end{array}$ & $\begin{array}{c}\text { Extensive } \\
\text { metabolizers } \\
\text { (EM) }\end{array}$ \\
\hline SLE & 60 & $7(11.7 \%)$ & $53(88.3 \%)$ \\
\hline Control & 129 & $12(9.3 \%)$ & $117(90.7 \%)$ \\
\hline $\begin{array}{l}\chi^{2}=0.253, p=0.615, p \text { - statistical significance }(p<0.05), \chi^{2}-\text { test } \chi^{2} \\
\text { comparison between } 2 \text { groups of patients with SLE and control subjects }\end{array}$
\end{tabular}

DNA was subjected to amplification using PCR with a pair of synthetic oligonucleotides, separately for the CYP2D6*3 and CYP2D6*4 mutations. PCR amplification was performed in a total reaction volume of $50 \mu \mathrm{l}$ containing 0.2-0.5 $\mu$ g genomic DNA, $1 U$ Taq Polymerase (Invitrogen USA), $1 \times$ PCR buffer (20 mM Tris- $\mathrm{HCl}$, pH 8.4, $50 \mathrm{mM} \mathrm{KCl}$ ), $1.5 \mathrm{mM} \mathrm{MgCl}$, $0.2 \mathrm{mM}$ of each dNTP (Fermentas, Germany), 5\% DMSO (Sigma, USA) and $250 \mathrm{nmol}$ of each primer (Oligo IBB PAN, Poland). PCR amplification was conducted in an Eppendorf Mastercycler Personal thermal cycler (Germany). The obtained product of amplification was subjected to digestion by restriction enzymes after control assessment in $2 \%$ agarose gel. The restriction endonucleases BstN 1 (New England BioLabs, USA) (CYP2D6*4) and Hpall (Invitrogen USA) (CYP2D6*3) were used to detect the presence or absence of mutations at the position G 1934 A and A 2637 deletion, respectively. Separation of the obtained digestion products was conducted using electrophoresis in $8 \%$ polyacrylamide gel. The obtained bands corresponding to particular DNA fragments following staining of the polyacrylamide gel with ethidium bromide were analysed in UV light.

The frequency distribution of CYP2D6 genotypes in SLE patients was compared with healthy subjects and analysed statistically using the $\chi^{2}$ test alone and with the Yates modification for small groups. Odds ratio (OR) with 95\% confidence interval
(95\% Cl) was calculated using the computer program Statistica 6.0.

\section{Results}

We determined whether there were any associations between susceptibility to SLE and particular CYP2D6 genotypes. Subjects were assigned to three different classes of overall genotypes (PM: CYP2D6*3/CYP2D6*4 or CYP2D6*4/ CYP2D6*4, heterozygous EM: CYP2D $6^{\star} 1 / C Y P 2 D 6^{\star} 4$ and homozygous EM: CYP2D $\left.6^{\star} 1 / C Y P 2 D 6^{\star} 1\right)$ on the basis of PCR results.

The frequency distribution of oxidation genotypes determining poor and extensive metabolizers in patients suffering from systemic lupus erythematosus and in controls is shown in Table I. There was no significant difference in the frequency of distribution of poor and extensive genotypes between patients with SLE and the control group.

The incidences of the three possible genotypes in the SLE population and in the controls as well as the OR SLE development are summarized in Table II. Among 60 patients with SLE, EM homozygotes of the $C Y P 2 D 6^{\star} 1$ allele with $C Y P 2 D 6^{\star} 1 / C Y P 2 D 6^{\star} 1$ genotype accounted for $45 \%$, and heterozygotes with CYP2D6*1/CYP2D6*4 genotype accounted for $41.67 \%$. Seven $(11.67 \%)$ people were PM with CYP2D6*3/CYP2D6*4 or CYP2D6*4/CYP2D6*4 genotype. Results obtained in the SLE group did not differ significantly from those in the control group.

Frequency of CYP2D6 gene alleles was compared between SLE patients and the control group. Differences in allele frequency between the groups were not statistically significant (Table III).

\section{Discussion}

The human organism is constantly exposed to harmful exogenous factors (xenobiotics) including drugs and carcinogenic compounds that can induce development of a large number of diseases. The

Table II. Frequency distribution of homozygous EM and PM and heterozygous EM patients with SLE and controls

\begin{tabular}{|c|c|c|c|c|c|c|c|}
\hline \multirow[t]{2}{*}{ CYP2D6 } & & \multicolumn{2}{|c|}{ Patients with SLE $(n=60)$} & \multicolumn{2}{|c|}{ Control group $(n=129)$} & \multirow{2}{*}{$\begin{array}{l}\text { Value } \\
\text { of } p\end{array}$} & \multirow[t]{2}{*}{ OR $(95 \% \mathrm{Cl})$} \\
\hline & & $N$ & $\%$ & $N$ & $\%$ & & \\
\hline \multirow{5}{*}{$\begin{array}{l}\text { Extensive } \\
\text { metabolizers } \\
\text { (EM) }\end{array}$} & $\begin{array}{l}\text { Homozygous: } \\
\text { CYP2D6 } 6^{\star} 1 / C Y P 2 D 6^{\star} 1\end{array}$ & 27 & 45 & 74 & 57.4 & 1.112 & $0.61(0.32-1.12)$ \\
\hline & Heterozygous: & & & & & & \\
\hline & $C Y P 2 D 6^{\star} 1 / C Y P 2 D 6^{*} 3$ & 1 & 1.66 & 4 & 3.1 & 0.571 & $0.53(0.06-4.85)$ \\
\hline & $C Y P 2 D 6^{\star} 1 / C Y P 2 D 6^{*} 4$ & 25 & 41.67 & 39 & 30.2 & 1.122 & 1.65 (1.14-3.09) \\
\hline & Total: & 53 & 88.33 & 117 & 90.7 & 0.615 & $0.78(0.29-2.07)$ \\
\hline \multirow{3}{*}{$\begin{array}{l}\text { Poor } \\
\text { metabolizers } \\
\text { (PM) }\end{array}$} & $C Y P 2 D 6^{*} 3 / C Y P 2 D 6^{*} 4$ & 1 & 1.67 & 0 & - & 0.141 & - \\
\hline & CYP2D6*4/CYP2D6*4 & 6 & 10 & 12 & 9.3 & 0.879 & $1.08(0.34-3.44)$ \\
\hline & Total: & 7 & 11.67 & 12 & 9.3 & 0.615 & $1.29(0.48-3.46)$ \\
\hline
\end{tabular}


processes of biotransformation in the organism are multidirectional and xenobiotics can be transformed into active or inactive metabolites via the oxidative route. They can also be transformed into harmful compounds with a potentially pathogenic action. The enzymatic system associated with the liver microsomal fraction with cytochrome P-450 as its fundamental element is responsible for xenobiotic oxidation. At present, the role of cytochrome P-450 and its isoforms in regulating not only the processes of drug metabolism and immune reactions but also in activating toxic substances has aroused great attention in the literature. Cytochrome P-450 enzymes show genetic polymorphism, which contributes to marked interindividual differences in drug responses. Examinations of the oxidative genotype or phenotype have been performed in order to identify the oxidative polymorphisms [18, 19]. Differences among individuals in drug responses due to mutations in the CYP2D6 gene that is involved in the metabolism of xenobiotics, including a number of drugs with a wide therapeutic use, were discovered as early as in the 1970s. Genetically determined oxidative polymorphism can affect not only drug efficacy but may also be a factor predisposing to disease development.

In the present study we tried to answer the question whether any associations exist between genetically determined activity of CYP2D6 enzyme and SLE development in the Polish population. There are numerous reports demonstrating the role of oxidative polymorphism in predisposing towards development of tumour diseases, allergy or psychiatric and neurological syndromes. Studies evaluating a predisposition towards tumour diseases have been carried out for a long time and predominantly concern tumours of the lungs, genitourinary tract and prostate as well as myeloblastic leukaemia. Those studies demonstrate that subjects who are extensive or ultra-rapid metabolizers account for the majority of patients with tumour diseases [20-24]. Studies on oxidative polymorphism have also been conducted in patients with Parkinson's or Alzheimer's diseases; however, the results are conflicting [13, 25, 26].

There are few reports in the available literature that concern the role of xenobiotic metabolizing enzyme polymorphism in autoimmune diseases $[14,15,27]$. However, a report about the association of CYP2D6 gene with SLE is still unavailable in Poland. Systemic lupus erythematosus is a model autoimmune disease that is characterized by increased production of autoantibodies against different antigens. Contemporary medicine is able to alleviate the symptoms only to a limited extent, with no possibility of complete recovery. The reason for that is still insufficient knowledge on SLE
Table III. Frequency of CYP2D6 alleles in patients with SLE and controls

\begin{tabular}{|lccc|}
\hline Allele & CYP2D6*1 & CYP2D6*3 & CYP2D6*4 \\
\hline Patients with & $\begin{array}{c}80 \\
(66.6 \%)\end{array}$ & $\begin{array}{c}2 \\
(1.7 \%)\end{array}$ & $\begin{array}{c}38 \\
(31.6 \%)\end{array}$ \\
\hline SLE (\%) & $\begin{array}{c}191 \\
(74 \%)\end{array}$ & $\begin{array}{c}4 \\
(1.6 \%)\end{array}$ & $\begin{array}{c}63 \\
(24.4 \%)\end{array}$ \\
\hline Controls (\%) & 0.7 & 1.08 & 1.43 \\
OR (95\% Cl) & $(0.44-1.11)$ & $(0.19-5.96)$ & $(0.89-2.29)$ \\
\hline
\end{tabular}

aetiopathogenesis, but there is evidence for a crucial role of genetic factors besides immunomodulatory and environmental factors in the disease development.

The first reports concerning associations between oxidative genotype and SLE were published in 1998. A study that was conducted among 69 patients with SLE and 514 healthy Caucasian volunteers showed that the incidence of the CYP2D $6^{*} 4$ allele was significantly higher in the group of SLE patients compared with the control group $(28.3 \%$ and $18.6 \%$, respectively). However, the difference was not statistically significant. It has been observed that the CYP2D6*4 allele occurred more frequently in SLE patients in whom renal involvement and haematological abnormalities had been established [15]. CYP2D6 polymorphism was also the subject of a study by Kortunay et al. However, the authors failed to show any association between the CYP2D6 genotype and the risk of developing SLE [14].

In our own studies we observed a higher proportion of subjects who were characterized by poor metabolism among SLE patients in comparison with the healthy ones. It has been shown that the frequency of genotypes for the poor and extensive oxidation in the group of SLE patients reached $11.7 \%$ and $88.3 \%$ respectively compared with $9.3 \%$ for poor metabolizers and $90.7 \%$ for extensive metabolizers in the control group. No statistically significant differences in the incidence of CYP2D6 genotypes for poor and extensive oxidation between the studied groups were found $(p=0.615)$. CYP2D $6^{\star} 1 / C Y P 2 D 6^{\star} 1$ and CYP2D6*1/CYP2D6*4 genotypes occurred most frequently in the group of SLE patients, whereas CYP2D $6^{*} 1 / C Y P 2 D 6^{\star} 1$ genotype was predominant among the controls. Relative risk of developing SLE, expressed as the odds ratio (OR), was not statistically significantly higher for the carriers of the particular genotypes.

The wild-type allele CYP2D6* 1 was found most frequently $(66.6 \%)$ in the group of patients with SLE. Both mutant alleles, i.e. CYP2D $6^{\star} 3$ and CYP2D $6^{*} 4$, accounted for $1.7 \%$ and $31.6 \%$, respectively, and these values were not statistically 
significantly different from the results obtained both in the control group $(74 \%, 1.6 \%$ and $24.4 \%$ respectively) and the results in the Caucasian population (70\%, $2 \%$ and $20 \%$ respectively) $[23,28$, 29]. Relative risk of SLE development was 1.08 -fold higher for the carriers of the CYP2D $6^{\star} 3$ allele and 1.43-fold higher for the subjects with the CYP2D6*4 allele; however, the risk was not statistically significantly higher. Increased occurrence of mutant alleles of the CYP2D6 gene in SLE patients and the calculated OR values could suggest the effect of these mutations on increased SLE development. Continuing the studies in a larger group of SLE patients is likely to provide confirmation of the observations and a more precise insight into the nature of the relationships between genetically determined metabolic factors and SLE development.

There are reports in the literature on the impact of the polymorphisms of other cytochrome P-450 enzymes on the risk of SLE development. Results obtained from the studies on the CYP1A1 gene implicate that the presence of polymorphisms in this gene might be a risk factor for SLE development and a predictor of renal involvement [30].

Knowledge of the genetically conditioned differences in oxidation processes as well as determination of the incidences of the principal mutations in the cytochrome P-450 isoenzyme CYP2D6 gene seems to be a vital clinical issue. Pharmacogenetic studies provide the possibility of establishing one of the potential reasons for the risk of developing autoimmune diseases, including SLE. Results from the studies that have been conducted so far allow the statement that oxidative polymorphisms are not a risk factor for developing SLE.

However, it seems that determination of CYP2D6 genotype in SLE patients is of significant clinical value since SLE is a disease that involves many organs and the treatment may require application of drugs that are subject to oxidation. Therapeutic effects with drugs subjected to oxidation are less satisfactory in extensive metabolizers compared with persons who are poor metabolizers. Relapses occur more frequently and remissions are quite rare among subjects who are extensive metabolizers. Treatment seems more effective in poor metabolizers but serious side effects that are associated with applying standard doses of drugs are frequently observed.

An examination of the oxidative genotype or phenotype allows individualization of pharmacotherapy. Knowledge of the oxidative polymorphism and its relationship with SLE may contribute to a safer and more efficient therapy since the disease is multiorgan and often requires drugs that belong to a variety of therapeutic groups.

\section{Acknowledgments}

This work was supported by grant No. 503-8011-1 from the Medical University of Lodz, Poland.

\section{References}

1. Sanchez E, Sabio JM, Callejas J, et al. Association study of genetic variants of pro-inflammatory chemokine and cytokine genes in systemic lupus erythematosus. BMC Medical Genetics 2006; 7: 48-55.

2. Nambiar MP, Juang YT, Krishanan S, Tsokos GC. Dissecting the molecular mechanisms of TCR $\zeta$ chain downregulation and $T$ cell signaling abnormalities in human systemic lupus erythematosus. Int Rev Immunol 2004; 23: 245-63.

3. Von Schmiedeberg S, Fritsche E, Ronnau AC, et al. Polymorphisms of the xenobiotic-metabolizing enzymes CYP1A1 and NAT-2 in systemic lupus erythematosus. Adv Exp Med Biol 1999; 45: 145-52.

4. Skrętkowicz J, Skrętkowicz-Szarmach K, Rychlik-Sych M. Genetic factors in pathogenesis of lupus erythematosus. Reumatologia 2004; 42: 267-72.

5. Linsey A, Criswell MD. The genetic contribution to systemus lupus erythematosus. Bull NYU Hosp Jt Dis 2008; 66: 176-83.

6. Yu A, Kneller BM, Rettie AE, Haining RL. Expression, putification, biochemical characterization, and comparative function of human cytochrome P450 2 D6.1, 2D6.2, 2D6.10, and 2D6.17 allelic isoforms. J Pharmacol Exp Ther 2002; 303: 1291-300.

7. Perera FP, Deliang T, Brandt-Rauf $P$, et al. Lack of Associations among cancer and albumin adducts, ras p21 oncoprotein levels, and CYP1A1, CYP2D6, NAT1, and NAT2 in nested case-CONTROL study of lung cancer within the physicians' health study. Cancer Epidemiol Biomarkers Prev 2006; 15: 1417-9.

8. Rodriguez-Antona C, Ingelman-Sundberg M. Cytochrome P450 pharmacogenetics and cancer. Oncogene 2006; 25: 1679-91.

9. Ingelman-Sunberg $M$. Genetic polymorphisms of cytochrome P450 2D6 (CYP2D6): clinical consequences, evolutionary aspects and functional diversity. Pharmacogenom J 2005; 5: 6-13.

10. Dahl ML. Cytochrome P450 phenotyping/genotyping in patients receiving antipsychotics. Useful aid to prescribing? Clin Pharmacokinet 2002; 41: 453-47.

11. Ken-Ichi F. Cytochrome P450 and anticancer drugs. Curr Drug Metabol 2006; 7: 23-37.

12. Van Schaik RHN. Cancer treatment and pharmacogenetics of cytochrome P450 enzymes. Invest New Drugs 2005; 23: 513-22.

13. Gołąb-Janowska M, Honczarenko K, Gawrońska-Szklarz B, Potemkowski A. CYP2D6 gene polymorphism as a probable risk factor for Alzhaimer's disease and Parkinson's disease with dementia. Neurol Neurochir Pol 2007; 41: 113-21.

14. Kortunay S, Bozkurt A, Bathum L, et al. CYP2D6 polymorphism in systemic lupus erythematosus patients. Eur J Clin Pharmacol 1999; 55: 21-5.

15. Sabbagh N, Marez D, Queyrel V, et al. Genetic analysis of cytochrome P450 CYP2D6 polymorphism in patients with systemic lupus erythematosus. Pharmacogenetics 1998; 8: 191-4.

16. Hochberg MG. Updating the American College of Rheumatology revised criteria for the classification of systemic lupus erythematosus (letter). Arthritis Rheum 1997; 40; 1725-8. 
17. Smith CA, Gough AC, Leigh PN, et al. Debrisoquine hydroxylase gene polymorphism and susceptibility to Parkinson's disease. Lancet 1992; 339: 1375-7.

18. Kot M, Daniel WA. Caffeine as a marker substrate for testing cytochrome P-450 activity in human and rat. Pharmacol Reports 2008; 60: 789-97.

19. Hosseini M, Houshmand M, Ebrahimi A. Breast cancer risk only was not assaciated with CYP17/A2 allele but also was related to A1 allele. Ach Med Sci 2009; 5: 103-6.

20. Ledesma MC, Agundez JAG. Identification of subtypes of CYP2D gene rearrangements among carriers of CYP2D6 gene deletion and duplication. Clin Chem 2005; 51: 939-43.

21. Sobti RC, Onsory K, Al-Badran Al, et al. CYP17, SRD5A2, CYP1B1, and CYP2D6 gene polymorphisms with prostate cancer risk in North Indian population. DNA Cell Biol 2006; 5: 287-94.

22. Sobti RC, Al-Badran Al, Sharma S, Sharma SK, Krishan A, Mohad H. Genetic polymorphisms of CYP2D6, GSTM1, and GSTT1 genes and bladder cancer risk in North India. Cancer Genet Cytogenet 2005; 156: 68-73.

23. Łapiński Ł, Agundez JAG, Wiela-Hojeńska A, et al. CYP2D6 gene amplification and the risk of acute myeloblastic leukemia. Pharmacol Rep 2007; 59 suppl. 1: 167-72.

24. Niewiński P, Orzechowska-Józwenko K, Hurkacz M, et al. CYP2D6 extensive, intermediate, and poor phenotypes and genotypes in a Polish population. Eur J Pharmacol 2002; 58: 533-5.

25. Yamada H, Dahl M, Viitanen M. No association between familial Alzheimer disease and cytochrome P450 polymorphism. Alzheimer Dis Assoc Disord 1998; 12: 204-7.

26. Woo S, Kim JW, Seo HG. Cyp2D6*4 polymorphism is not associated with Parkinson's disease and has no protective role against Alzheimer's disease in the Korean population. Psych Clin Neurosci 2001; 55: 373-7.

27. Rychlik-Sych M, Skrętkowicz J, Gawrońska-Szklarz B, Górnik W, Sysa-Jędrzejowska A, Skrętkowicz-Szarmach K. Acetylation genotype and phenotype in patients with systemic lupus erythemotosus. Pharmacol Rep 2006; 58: 22-9.

28. Xie HG, Kim RB, Wood AJJ, Stein CM. Molecular basis of ethnic differences in drug disposition and response. Ann Rev Pharmacol Toxicol 2001; 41: 815-50.

29. Fukuda T, Maune H, Ikenaga Y, Naohara M, Fukuda K, Azuma J. Novel structure of the CYP2D6 gene that confuses genotyping for the CYP2D6 5 allele. Drug Met Pharmacokinet 2005; 20: 345-50.

30. Yen JH, Chen CJ, Tsai WC, et al. Cytochrome P450 and manganese superoxide dismutase genes polymorphisms in lupus systemic erythematosus. Immunol Lett 2003; 90: 19-24. 